\title{
DIAGNÓSTICO PRENATAL DE LAS MALFORMACIONES PULMONARES CONGÉNITAS. REPORTE DE UN CASO
}

\author{
PRENATAL DIAGNOSIS OF CONGENITAL LUNG MALFORMATIONS: REPORT OFA CASE \\ DIAGNOSTICO PRÉ-NATAL DAS MALFORMAÇÕES PULMONARES CONGÉNITAS: REPORTE DE UM CASO
}

\author{
CARLOS ANDRÉS PEÑAHERRERA OVIEDO', MARÍA CAROLINA DUARTE MARTÍNEZ1 \\ ${ }^{1}$ Universidad Católica de Santiago de Guayaquil, Ecuador
}

Resumen

Las malformaciones pulmonares congénitas son el resultado de diferentes tipos de trastornos en el desarrollo del árbol traqueobronquial, durante la vida embrionaria. A pesar de tener una baja incidencia, sus complicaciones pueden resultar determinantes para la sobrevida tanto a nivel prenatal como postnatal. Los tipos más frecuentes de malformaciones pulmonares son la malformación adenomatoidea quística y el secuestro broncopulmonar. El control durante el embarazo y la realización de ultrasonido permite el diagnóstico temprano, el estudio individualizado y la decisión terapéutica a seguir. Se debe siempre realizar el análisis histopatológico para determinar el riesgo de malignidad. Se presenta el caso de un paciente lactante de sexo masculino a quien se le detectó prenatalmente masa quística en hemitórax izquierdo; fue manejado quirúrgicamente a los 4 meses del nacimiento. En este tipo de casos, el conocimiento del tipo de malformación, su tamaño, compromiso respiratorio y posible manejo, resulta vital para el equipo interdisciplinario encargado del paciente, para asegurar un mejor pronóstico y calidad de vida.

PALABRAS CLAVE: Secuestro broncopulmonar, neonatología, diagnóstico diferencial.

Abstract

Congenital Lung Malformations are the result of different types of disorders in the development of the tracheobronchial tree during embryonic life. Despite having a low incidence, complications may result into determinants for survival at prenatal and postnatal levels. The most frequent types of Lung malformations are congenital cystic adenomatoid malformation and bronchopulmonary sequestration. Control during pregnancy and ultrasound allow early diagnosis, individualized study, and therapeutic decision to be made. It is always necessary to perform histopathological examination to determine malignancy risk. A case of a lactating patient is presented. The patient was prenatally diagnosed with cystic mass on left hemithorax; it was treated surgically at the age of 4 moths. In this type of cases, knowledge of the type of malformation, size, respiratory problems and possible treatment is vital for the interdisciplinary team in charge of the patient to assure better prognosis and quality of life.

KEYWORDS: bronchopulmonary sequestration, neonatology, diagnosis differential.

Resumo

As malformações pulmonares congénitas são o resultado de diferentes tipos de transtornos no desenvolvimento da arvore traqueobrônquica, durante a vida embrionária. A pesar de ter uma baixa incidência, as suas complicações podem resultar determinantes para a sobrevida tanto ao nível pré-natal como pós-natal. Os tipos mais frequentes de malformações pulmonares são a malformação adenomatoidea quistica e o sequestro bronco pulmonar. 0 controle durante a gravidez e a realização de ultrassom permite o diagnostico prematuro, o estudo individualizado e a decisão terapêutica a seguir. A analise histopatológica sempre deve ser feita para determinar o risco de malignidade. 0 caso de um paciente lactante de sexo masculino e apresentado no qual se detectou prenatalmente uma massa quistica no hemotórax esquerdo; foi dirigido cirurgicamente aos 4 meses depois do nascimento. Neste tipo de casos, 0 conhecimento do tipo de malformação, o tamanho, comprometimento respiratório e possível manejo, resulta vital para o time interdisciplinar encarregado do paciente, para segurar um melhor prognostico e qualidade de vida.

PALABRAS-CHAVE: sequestro broncopulmonar, neonatologia, diagnóstico diferencial. 
INTRODUCCIÓN

Las malformaciones pulmonares congénitas son un grupo heterogéneo de trastornos del desarrollo del árbol traqueobronquial. ${ }^{1}$ Son poco frecuentes, con una incidencia de entre 1:25000 y 1:35000, y representan del 15 a $50 \%$ de las enfermedades quísticas congénitas del pulmón. , $^{2,3} \mathrm{El}$ espectro clínico es variado, desde malformaciones asintomáticas, hasta malformaciones que causan severo distrés respiratorio pre y postnatal, o incluso son incompatibles con la vida. ${ }^{4}$ Entre los principales tipos de malformaciones pulmonares congénitas encontramos el enfisema lobar congénito, el quiste broncogénico, la malformación adenomatoidea quística (MAQ), el secuestro pulmonar intra y extralobar, el síndrome de la cimitarra, y las malformaciones arteriovenosas. ${ }^{1}$

En ocasiones se realiza el diagnóstico prenatal de las malformaciones pulmonares por medio del ultrasonido, lo que ha venido a mejorar el pronóstico de estos pacientes. ${ }^{5,6} \mathrm{~A}$ pesar de no poder determinar específicamente el tipo de malformación, nos facilita datos preliminares acerca de la composición de la masa. ${ }^{7}$ La resonancia magnética nuclear (RMN) ha surgido como soporte en el diagnóstico prenatal debido a que es inocua para el feto, y muestra con detalle las masas intratorácicas. ${ }^{7}$ En el caso de requerir el uso de radiografías o tomografía computarizada (TC), deberá sopesarse el riesgo-beneficio. ${ }^{6,7}$

Debido a que este tipo de malformaciones pueden tener potencial maligno, debe evaluarse cada caso particular de manera interdisciplinaria con el fin de decidir el mejor paso terapéutico, ya sea la resección quirúrgica o el manejo conservador. ${ }^{2,8}$ El reporte histopatológico da el diagnóstico final, y en ocasiones se observa la coexistencia de dos o más tipos distintos de malformación. ${ }^{9,10} \mathrm{~A}$ continuación presentamos el caso de un paciente masculino en quien se realizó prenatalmente el diagnóstico de una masa quística intratorácica, la cual fue removida a los 4 meses de edad sin complicaciones, y donde el estudio patológico reportó un secuestro pulmonar con MAQ coexistente.

PRESENTACIÓN DEL CASO

Paciente masculino de 4 meses y medio fue traído para valoración quirúrgica de una masa pulmonar detectada de manera prenatal. El paciente es producto del segundo embarazo de una madre aparentemente sana de 28 años de edad. Quien cursó una gestación normoevolutiva con adecua- dos controles prenatales y ecográficos hasta la semana 28 de gestación, donde un ultrasonido de rutina detectó una masa ecogénica con zonas anecogénicas en la base del pulmón izquierdo. (Figura 1).

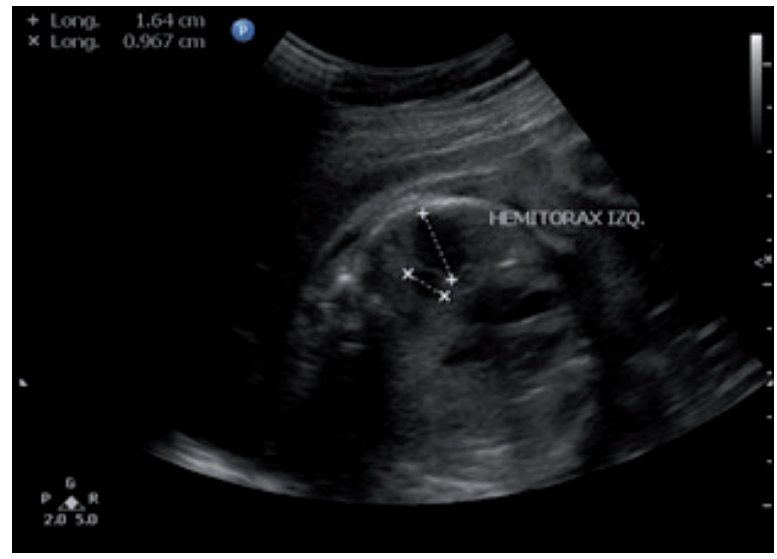

Figura 1. Ultrasonido fetal donde se observa masa ecogénica en base pulmonar izquierda, con múltiples contenidos anecogénicos.

Para determinar la naturaleza de dicha masa se solicitó resonancia magnética prenatal, que mostró una lesión multiquística intrapulmonar en base izquierda, hipointensa en secuencia T1 e hiperintensa en T2, y se obtuvo un diagnóstico presuntivo de malformación adenomatoidea quística (MAQ) tipo 1. (Figura 2). Se realizó sesión interdisciplinaria para decidir el manejo de la masa pulmonar, donde se determinó realizar ultrasonidos cada dos semanas para seguimiento de la misma, además de aplicar esquema de maduración pulmonar con betametasona.

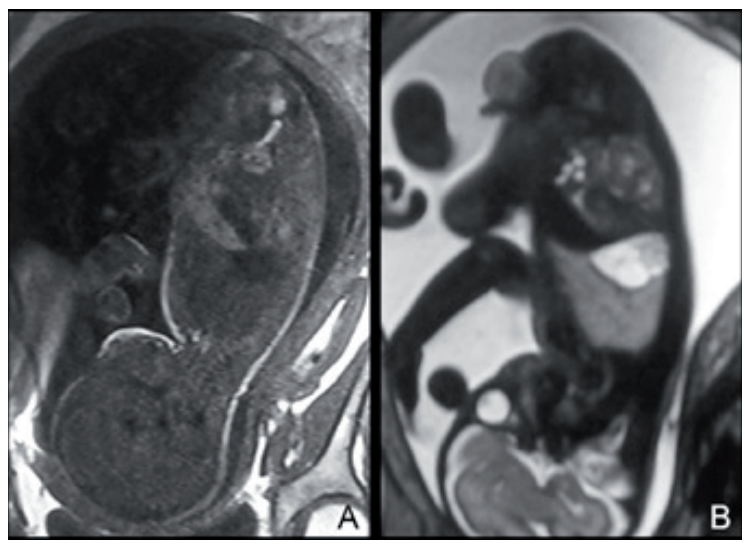

Figura 2. RMN prenatal a las 28 semanas: A) Secuencia T1, se observa masa hipointensa en base pulmonar izquierda, sus dimensiones son $2.3 \mathrm{x}$ $3.2 \times 2 \mathrm{~cm}$. B) Secuencia T2 donde se muestra hiperintensidad heterogénea en la misma localización. El resto de la anatomía es normal.

Los ultrasonidos subsecuentes no mostraron evolución ni involución de la masa. En la semana 38 se realizó cesárea segmentaria por presencia de estreptococo grupo B en canal vaginal. Se 
obtuvo producto único masculino de $3.35 \mathrm{Kg}$ de peso, que mide 51.5 centímetros, APGAR $9 / 9$ al minuto y a los 5 minutos respectivamente, de 38 semanas gestacionales según escala de Capurro. Los exámenes de laboratorio fueron normales a excepción de leucocitosis de 18.200 cél/uL, con 67\% de neutrófilos, y procalcitonina de 17.66 $\mathrm{ng} / \mathrm{ml}$. El tamizaje neonatal fue normal. Para continuar la evaluación de la masa pulmonar, se realizó radiografía de tórax que sólo mostraba ingurgitación pulmonarbilateral, yuna tomografía de tórax con contraste intravenoso, donde se evidenció una masa hipodensa en lóbulo inferior izquierdo, que no realzaba con contraste. (Figura 3). Un nuevoultrasonido torácico mostró una masa multiquística similar a la observada durante el embarazo. El paciente egresó 3 días después del nacimiento, sin datos de dificultad respiratoria ni de respuesta inflamatoria sistémica. Se planificó seguimientos subsecuentes con cirugía pediátrica para agendar la extracción de la masa pulmonar en una edad más avanzada.
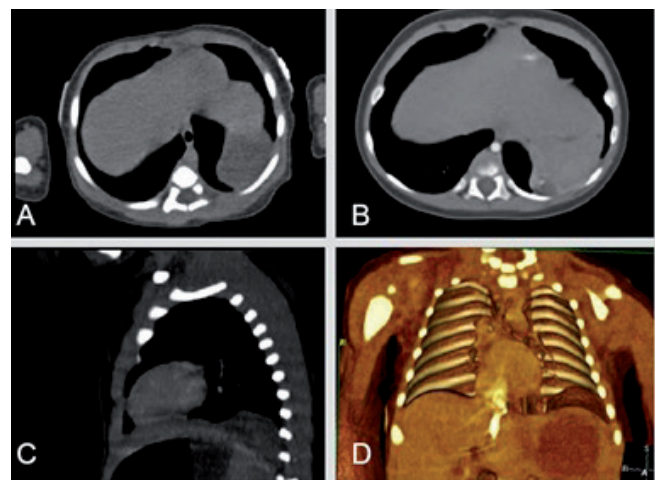

Figura 3. TC de tórax postnatal. A) Corte transversal donde se observa masa hipodensa en base pulmonar izquierda, mide $24 \times 27 \mathrm{~mm}$. B) Luego de la administración de contraste IV, la masa muestra una hiperdensidad central puntiforme, que insinúa la presencia de vaso nutricio. C) Vista lateral, se observa la localización de la masa en fosa pleural izquierda. D) Reconstrucción $3 \mathrm{D}$ donde se observa claramente la masa intratorácica.

Se programó cirugía de resección a los 4 meses y medio de edad, con diagnóstico preoperatorio de MAQ vs. secuestro pulmonar. Las pruebas de laboratorio antes de la cirugía fueron normales. Se realizó toracotomía posterolateral izquierda por planos, con retracción de pulmón izquierdo, tras lo cual se encontró una masa de parénquima pulmonar adherida a diafragma, no conectada a vía aérea y con vaso nutricio proveniente de aorta abdominal, descripción compatible con secuestro pulmonar extralobar. Tras la disección de la misma y la ligadura del vaso arterial y sección del mismo, se realizóresección dela masa deaproximadamente $3 \mathrm{x}$ $3 \mathrm{~cm}$. (Figura 4). Posteriormente se reforzó la cúpula diafragmática con un punto de sutura y se realizó cierre por planos y aspiración con sonda. El paciente ingresó a posoperatorio hemodinámicamente estable; la saturación de oxígeno bajó a $80 \%$, y luego recuperóa $100 \%$, con oxígeno por casco. Se mantuvo con tratamientoantibiótico con cefalotina $270 \mathrm{mg}$ IV c/8h y analgesia. Al día siguiente la radiografía de control no muestra alteraciones, por lo que se retiró oxígeno suplementario y el paciente mantuvo una saturación normal, hemodinámicamente estable. Al segundo día de postoperatorio se otorgó el alta médica, con orden para controles subsecuentes por consulta externa.

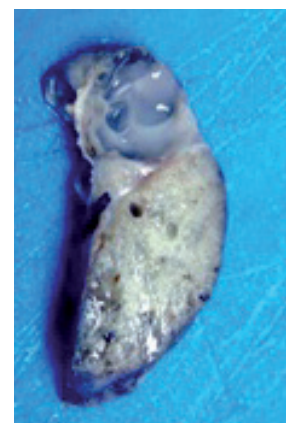

Figura 4. Corte de la masa pulmonar extirpada, donde se observa una estructura mixta, con un componente sólido y otro quístico. Dimensiones: $3.2 \times 2 \times 1 \mathrm{~cm}$.

En el servicio de patología se recibió pieza de parénquima pulmonar de consistencia sólida que midió $3.3 \times 2 \times 1 \mathrm{~cm}$. Al corte, se observó una masa sólida que contenía múltiples quistes pequeños con contenido mucoso claro y brillante. El examen histopatológico reveló la presencia de tejido pulmonar con dilatación de quistes bronquiales y escaso desarrollo alveolar, compatible histológicamente con secuestro pulmonar. La presencia de múltiples quistes con contenido de moco bronquial y de proliferación glandular añade el diagnóstico de MAQ tipo 3 asociada al secuestro pulmonar. (Figura 5).
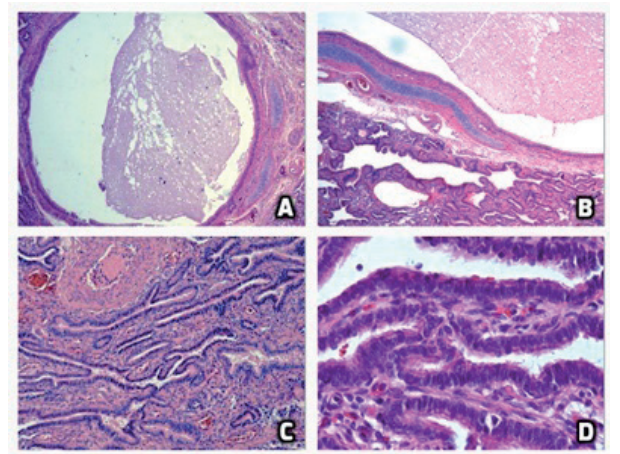

Figura 5. Histopatología con H\&E. A) Aumento 4x donde se observa bronquio dilatado, con material mucinoso en su interior. B) Aumento 10x, se evidencia tejido bronquial normal con epitelio cilíndrico ciliado, submucosa, y cartílago (compatible con SBP), junto a una zona de proliferación glandular. C) Aumento 10x, se observa proliferación glandular desorganizada. D) Aumento 40x, donde es notoria la presencia de epitelio cúbico glandular, compatible con MAQ tipo 3. 
DISCUSIÓN

Las malformaciones pulmonares congénitas corresponden a una amplia gama de trastornos del desarrollo del aparato respiratorio, que a pesar de su baja incidencia, pueden impactar de manera negativa la calidad de vida y el pronóstico de los neonatos. ${ }^{1,2,6}$ Estas malformaciones seclasifican de acuerdo a su composición histológica, y sus manifestaciones clínicas van desde la presentación asintomática hasta la completa incompatibilidad con la vida. ${ }^{4}$ Los tipos más frecuentes de malformaciones pulmonares son la malformación adenomatoidea quística (MAQ) y el secuestro broncopulmonar. ${ }^{6}$

Las MAQ corresponden a la variante más frecuente de las malformaciones congénitas del árbol respiratorio, y a su vez se subdividen en 5 grupos dependiendo de las características histológicas de la mucosa. (Tabla 1).2,6,11

\begin{tabular}{|c|c|c|c|}
\hline $\begin{array}{l}\text { Tipo de } \\
\text { MAQ }\end{array}$ & $\begin{array}{l}\text { Características } \\
\text { histopatológicas }\end{array}$ & $\begin{array}{l}\text { Preva- } \\
\text { lencia }\end{array}$ & $\begin{array}{l}\text { Complicaciones } \\
\text { asociadas }\end{array}$ \\
\hline 0 & $\begin{array}{l}\text { Displasia/agenesia acinar. } \\
\text { Compromiso multilobar. }\end{array}$ & $<2 \%$ & Muerte fetal \\
\hline 1 & $\begin{array}{l}\text { Quiste único o múltiples, } \\
>2 \mathrm{~cm} \text {. Epitelio columnar } \\
\text { pseudoestratificado. }\end{array}$ & $\begin{array}{l}60- \\
70 \%\end{array}$ & $\begin{array}{l}\text { Carcinoma bronquioloal- } \\
\text { veolar }\end{array}$ \\
\hline 2 & $\begin{array}{l}\text { Quiste único o múltiples, } \\
<2 \mathrm{~cm} \text {. Epitelio cuboidal o } \\
\text { columnar. }\end{array}$ & $\begin{array}{r}15- \\
20 \%\end{array}$ & $\begin{array}{l}\text { Malformaciones de otros } \\
\text { sistemas, principalmente } \\
\text { genitourinario. Rabdomio- } \\
\text { sarcoma. }\end{array}$ \\
\hline 3 & $\begin{array}{l}\text { Lesiones predominantemente } \\
\text { sólidas, quistes de }<0.5 \mathrm{~cm} \text {, } \\
\text { epitelio cuboidal }\end{array}$ & $\begin{array}{r}5- \\
10 \%\end{array}$ & $\begin{array}{l}\text { Polihidramnios, hidrops. } \\
\text { Atresia bronquial. }\end{array}$ \\
\hline 4 & $\begin{array}{l}\text { Quistes grandes llenos de } \\
\text { aire, epitelio plano. Distribu- } \\
\text { ción periférica }\end{array}$ & $<10 \%$ & $\begin{array}{l}\text { Lesiones hamartomato- } \\
\text { sas. Múltiples neoplasias }\end{array}$ \\
\hline
\end{tabular}

Se caracteriza por la presencia de múltiples masas quísticas en el parénquima pulmonar, como resultado de la proliferación anormal de los bronquiolos terminales, y supresión del desarrollo alveolar entrela semana 7 y 15 de gestación. ${ }^{12}$ Estas lesiones hamartomatosas son usualmente asintomáticas al nacimiento, sin embargo en ciertos casos se presenta distrés respiratorio progresivo y severo debido a la expansión de los quistes; esto llega en ocasiones a producir hidrops fetal. ${ }^{2}$ En ocasiones, las MAQ ocasionan trastornos ventilatorios restrictivos, que pueden detectarse mediante pruebas de función pulmonar. ${ }^{3,13}$ El análisis histopatológico es importante para el pronóstico del paciente, ya que la determinación del subtipo de MAQ determina el riesgo de malignidad (carcinoma bronquialveolar en las MAQ tipo 1), alteraciones asociadas del sistema genitourinario (MAQ tipo 2), o atresia laríngea (en casos de MAQ tipo 3). ${ }^{9}$

El secuestro broncopulmonar (SBP) es el segundo tipo más frecuente de malformación pulmonar congénita, y corresponde a un segmento de tejido pulmonar normal no conectado con el resto del árbol bronquial. ${ }^{10}$ Se piensa que se originan de un brote accesorio del árbol traquebronquial durante las primeras semanas del desarrollo. ${ }^{4}$ Se identifican dos tipos de SBP: el intralobar, que comparte la pleura con el resto del pulmón adyacente, y el extralobar, que tiene su propia cubierta pleural. ${ }^{5}$ Estos últimos son más frecuentes en el hemitórax izquierdo (48\%), como sucedió en el caso antes descrito. ${ }^{9}$ Los SBP suelen tener un vaso nutricio originado en una arteria extratorácica, como la aorta abdominal, ${ }^{4}$ y pueden contener quistes y bronquiolos dilatados debido a la producción de moco por la mucosa normal, que no puede ser drenado alárbol respiratorio. ${ }^{9}$ Con relativa frecuencia, los SBP coexisten con la presencia de una MAQ en su interior, lo que coincide con lo observado en nuestro paciente. ${ }^{10}$

El diagnóstico prenatal de las malformaciones pulmonares en el feto se da inicialmente por el ultrasonido de control, donde pueden observarse imágenes ecogénicas con componentes quísticos en un lado del tórax. ${ }^{5,14}$ Si bien mediante ultrasonido no es posible diferenciar entre MAQ, SBPu otros tipos de malformaciones, aporta datos importantes en cuantoal tamaño aproximado dela masa yal compromiso respiratorio, además de hacer posible la visualización de casos de hidrops. ${ }^{6,15}$ Por otro lado, al utilizar doppler con color puede detectarse la presencia de anomalías vasculares asociadas a la malformación..$^{6,7}$ La radiografía de tórax se utiliza para los controles postnatales, mientras que la TC nos provee de datos exactos acerca del tamaño, localización, eirrigación de la masa (en caso de utilizar contraste). ${ }^{7,16} \mathrm{La}$ TC durante el embarazo, por otro lado, se reserva para casos de fetos sintomáticos o donde se requiera intervención quirúrgica intrauterina, ya que los beneficios superan a los riesgos de la radiación., ${ }^{1,7}$ La RMN con frecuencia complementa al ultrasonido en el diagnóstico prenatal, pues muestra con detalle las dimensiones y localización de la masa y no emite radiación, sin embargo requiere un mayor tiempo para el estudio, donde los movimientos fetales pueden alterar la imagen, por lo que con frecuencia es necesaria la sedación con la morbilidad fetal asociada. El manejo de este tipo de malformaciones siempre dependerá del estado clínico del producto. 
La mayoría de las malformaciones son asintomáticas al nacimiento y, en el caso de los SBP, suelen involucionar conforme el feto se acerca al término del embarazo., ${ }^{417}$ Ante la presencia de hidrops fetal, está indicada la betametasona, y en caso de no lograrse regresión se debe realizar intervención quirúrgica intrauterina o inducir un parto pretérmino. ${ }^{8,17}$

La mortalidad fetal de la cirugía prenatal en estos casos es de alrededor del 50\%. ${ }^{14}$ Alrededor del 10\% de los pacientes presentarán síntomas en la etapa neonatal, por lo que se practica la resección quirúrgica. ${ }^{18}$ La presencia de un patrón respiratorio restrictivo apoya la decisión de manejo quirúrgico. ${ }^{13}$ En aquellos pacientes asintomáticos, en caso de que la lesión no involucione, se prefiere realizar cirugía electiva antes de que empiece a presentar síntomas, por lo que se suele diferir la cirugía hasta después de los tres meses del nacimiento, cuando el lactante está más apto para tolerar una cirugía de una magnitud como la toracotomía o la toracoscopía. ${ }^{17,18}$ En estos casos, la sobrevida es excelente, superior al $98 \% .{ }^{19}$ En presencia de una lesión pulmonar única y asintomática, podría sugerirse el manejo conservador, pues no se ha determinado si el riesgo de complicaciones supera al riesgo quirúrgico en estos pacientes. ${ }^{20}$ Toda masa pulmonar extirpada debe ser enviada a patología para el correspondiente examen histopatológico, que da el diagnóstico definitivo y proporciona datos con respecto al pronóstico. ${ }^{9}$

\section{CONCLUSIONES}

Como se pudo observar en el presente caso, el diagnóstico prenatal de las malformaciones pulmonares congénitas permite anticipar las medidas terapéuticas y diseñar un plan adecuado para cada paciente. El abordaje debe ser interdisciplinario y buscar la medida que más beneficie al individuo. En nuestro caso, el paciente tuvo un curso asintomático y no complicado, por lo que pudo realizarse la resección quirúrgica sin inconvenientes posteriores. Sin duda lo más curioso del paciente presentado es la coexistencia de una MAQ dentro del secuestro pulmonar, lo que al compararlo con datos históricos de cohortes de pacientes con hallazgos similares, hace notar que esta correlación es más frecuente de lo inicialmente descrito. ${ }^{10}$

\section{REFERENCIAS BIBLIOGRÁFICAS}

1. Lee EY, Dorkin H, Vargas SO. Congenital Pulmonary Malformationsin Pediatric Patients: Review and Update onEtiology, Classification, and Imaging Findings. Radiol Clin. 2011 Sep 1;49(5):921-48.
2. BoldeS, PudaleS, Pandit G, RuikarK, IngleSB. Congenital pulmonaryairway malformation: A report of two cases. World J Clin Cases. 2015 May 16;3(5):470-3.

3. Kittyle MK, Miranda RC, GonzálezMG, Fariña DA. Malformación adenomatoidea quística. Caso reportadoenel hospital pediátrico “Dr. Roberto GilbertElizalde”, terapia intensiva neonatal. Rev Med. 2009 Apr 30;14(3):242-6.

4. Laberge J-M, Puligandla P, Flageole H. Asymptomatic congenital lung malformations. Semin Pediatr Surg. 2005 Feb;14(1):16-33.

5. Zozzaro-SmithP. Ultrasonography for FetalLung Masses. Ultrasound Clin. 2013 Jan 1;8(1):49-54.

6. Gajewska-Knapik K, Impey L. Congenital lung lesions: Prenataldiagnosisand intervention. Semin PediatrSurg. 2015 Aug;24(4):156-9.

7. Chowdhury MM, Chakraborty S. Imaging of congenital lung malformations. Semin Pediatr Surg. 2015 Aug;24(4):168-75.

8. ParikhDH, RasiahSV. Congenitallunglesions: Postnatal management and outcome. Semin Pediatr Surg. 2015 Aug;24(4):160-7.

9. Fowler DJ, Gould SJ. The pathology of congenital lung lesions. Semin Pediatr Surg. 2015 Aug;24(4):176-82.

10. Conran RM, Stocker JT. Extralobar sequestration with frequently associated congenital cystic adenomatoid malformation, type 2: report of 50 cases. Pediatr Dev Pathol Off J Soc Pediatr Pathol Paediatr Pathol Soc. 1999 Oct;2(5):454-63.

11. Stocker JT. Cystic lung disease in infants and children. Fetal Pediatr Pathol. 2009;28(4):155-84.

12. Hasnain Fatimi S, Asif Ali T, Majid Z, Deedar Ali R. Congenital cystoid adenomatoid malformation: Surgery in a young child. Asian Cardiovasc Thorac Ann. 2015 Jul;23(6):735-7.

13. Barikbin P, RoehrCC, WilitzkiS, KalacheK, Degenhardt $\mathrm{P}$, Bührer C, et al. Postnatal lung function in congenital cysticadenomatoid malformation of thelung. AnnThorac Surg. 2015 Apr;99(4):1164-9.

14. Durell J, LakhooK. Congenital cystic lesions of the lung. Early Hum Dev. 2014 Dec;90(12):935-9.

15. Turkyilmaz A, Aydin Y, Erdem AF, Eroglu A, Karaoglanoglu N. Congenital Cystic Pulmonary Malformations in Children: Our Experience with 19 Patients. Eurasian JMed. 2009 Apr;41(1):15-21.

16. Zhang Z-J, Huang $M-X$. Children with congenital cystic adenomatoid malformation of thelung CTdiagnosis. Int JClin Exp Med. 2015 Mar 15;8(3):4415-9.

17. Baird R, Puligandla PS, Laberge J-M. Congenital lung malformations: informing best practice. Semin Pediatr Surg. 2014 Oct;23(5):270-7.

18. Singh R, Davenport $M$. The argument for operative approach to asymptomatic lung lesions. Semin Pediatr Surg. 2015 Aug;24(4):187-95.

19. WagenaarAE, TashiroJ, Hirzel A, RodriguezLI, PerezEA, Hogan AR, et al. Surgical management of bronchopulmonary malformations. J Surg Res. 2015 Apr 1;

20. Stanton $M$. The argument for a non-operative approach to asymptomaticlunglesions. Semin Pediatr Surg. 2015 Aug;24(4):183-6. 